\title{
Analysis of the correlation between Zeste enhancer homolog 2 (EZH2) mRNA expression and the prognosis of mesothelioma patients and immune infiltration
}

\section{Yun-chuan Sun ( $\nabla$ sunyunchuan@163.com )}

Cangzhou Hospital of Integrated TCM-WM Hebei

Kui Fan

Cangzhou Hospital of Integrated TCM-WM Hebei

Chuan-long Zhang

China Academy of Chinese Medical Sciences

Bo-hui Zhang

Cangzhou Hospital of Integrated TCM-WM Hebei

Meng-qi Gao

Shandong university of traditional Chinese medicine

\section{Research Article}

Keywords: EZH2, MESO, TCGA, Cell cycle, NK cells

Posted Date: February 7th, 2022

DOI: https://doi.org/10.21203/rs.3.rs-1295410/v1

License: (c) (1) This work is licensed under a Creative Commons Attribution 4.0 International License.

Read Full License 


\section{Abstract}

Background: Mesothelioma is one of the most malignant tumors, which makes the identification of mesothelioma biomarkers extremely important. To investigate the prognostic value of enhancer homolog 2 (EZH2) mRNA expression in mesothelioma patients and its immune infiltration analysis.

Methods: Gene expression and clinical information, enrichment analysis, and immune infiltration analysis obtained based on the Cancer Genome Atlas (TCGA) were performed, and additional bioinformatics analysis was performed. Clinical information and gene expression were obtained from 86 patients with mesothelioma based on the Cancer Genome Atlas TCGA database. Survival analysis, GSEA enrichment analysis, and immune infiltration analysis of $E Z H 2$ expression were performed by using R (version 3.6.3) (statistical analysis and visualization). Using the TIMER database (Fig. https://cistrome.shinyapps.io/timer/ ), the correlation between EZH2 expression and immune cell infiltration in mesothelioma was analyzed.

Results: We collated the data obtained from the TCGA database and performed univariate and multivariate analysis of general data including age, gender, stage, pathological type, and whether they had received radiotherapy, and the results showed that high expression of $E Z H 2$ was associated with poor prognosis in mesothelioma patients. The prognosis was worse in the High group (HR=2.75, 95\% Cl: 1.68-4.52, P冈0.010). Moreover, ROC curves showed that EZH2 expression predicted 1-year survival with an AUC of 0.740, 2-year survival with an AUC of 0.756, and 3-year survival with an AUC of 0.692, suggesting that $E Z H 2$ expression has a good predictive effect on prognosis. KEGG pathway analysis showed that there were five pathways with the strongest positive correlation with $E Z H 2$ expression: Cell cycle, DNA replication, Cell adhesion molecules cams, Primary immuno deficiency, Tsate transduction, and five pathways with the strongest negative correlation with EZH2 expression: Glycolysis gluconeogenesis , Drug metabolism, cytochrome $\mathrm{P} 450$, retinol metabolism, fatty acid metabolism ribosom. We analyzed the correlation between $E Z H 2$ expression and the level of immune infiltration in mesothelioma tissues. The results indicate that $E Z H 2$ expression plays an important role in immune infiltration. At high $E Z H 2$ expression, the number of NK cells, Mast cells and Th17 cells was reduced. Mesothelioma patients with high EZH2 expression differ from mesothelioma patients with low EZH2 expression in their tumor immune microenvironment.

Conclusion: $E Z H 2$, as a new prognostic biomarker for mesothelioma, is helpful to elucidate how changes in the immune environment promote the development of mesothelioma. Further analysis, $E Z H 2$ may be used as a biological test to predict the prognosis of mesothelioma.

\section{Introduction}

Mesothelioma is a malignant tumor arising from the mesothelial surface of the pleural cavity, peritoneal cavity, testicular tunica vaginalis, or pericardium, of which $80 \%$ of cases originate from the pleura[1]. Mesothelioma is a rare malignancy with a poor prognosis and an overall survival(OS) of approximately 9- 
17 months after diagnosis [1]. In particular, patients with pleural mesothelioma, because of its low incidence in mesothelioma, although there are now some related studies, chemotherapy, immunization, local radiotherapy, the effect is not obvious. The identification of molecular biomarkers predictive of prognosis is limited by the small number of mesothelioma patients who have joined clinical trials as well as the heterogeneity of the study population. Therefore, it is important to seek therapeutic targets for this malignancy.

Recent studies of $E Z H 2 \mathrm{mRNA}$ are in full swing, and its mechanism is associated with silencing epigenetic genes [2]. Studies have shown that abnormal expression of $E Z H 2$ is associated with poor prognosis in various malignancies such as lung[3]. EZH2 regulates gene expression to prevent differentiation of stem and progenitor cells, and abnormalities in EZH2 activity are thought to be drivers of carcinogenesis. And $E Z H 2$ inhibitor monotherapy showed good antitumor activity in the treatment of anti-mesothelioma, with good safety/tolerability [4].

However, EZH2 has been less studied with mesothelioma, and its role in mesothelioma is unknown. In this paper, based on the clinical data in the TCGA database, the expression of EZH2 was statistically analyzed for the prognosis of mesothelioma, and the correlation between $E Z H 2$ and immune infiltration in patients with mesothelioma was statistically analyzed in order to further elaborate the mechanism of EZH2s immune impact in patients with mesothelioma (tumor microenvironment) and seek the relevant impact mechanism. Our work revealed that increased expression of $E Z H 2$ is linked to poor OS among Mesothelioma patients. GSEA pathways showed that involved in Cell cycle, DNA replication, Primary immuno deficiency, Metabolism were associated with EZH2 expression. Moreover, we were able to identify a connection between $E Z H 2$ and tumor- infiltrating immune cells. The presented work here provides a detailed analysis of the role of $E Z H 2$ in Mesothelioma development, which will aid in the understanding of the underlying mechanisms Mesothelioma.

\section{Materials And Methods}

\subsection{Evidence from TCGA database}

We based on TCGA (https://portal.gdc.cancer.gov). The database was obtained from the MESO (mesothelioma) project with immune system infiltration, gene expression (HTSeq-FPKM) and related clinical information[5]. Samples with missing or insufficient data in terms of age, TNM stage, OS time, distant and lymph node metastasis were excluded from our study. RNA-Seq and clinical data were retained for further study. Our study conformed to the publication guidelines provided by TCGA.

\subsection{Survival Analysis}

Based on the clinical information obtained from the TCGA database, the relationship between EZH2 expression (high and low expression in median) and mesothelioma patient survival was analyzed by using the software: R (version 3.6.3) (statistical analysis and visualization) R package: survminer 
package [0.4.9 version] (for visualization), survival package [3.2-10 version] (for statistical analysis of survival data); survival curves were plotted. Statistical criteria were: Pख0.05.

\subsection{GSEA enrichment analysis}

We performed GSEA using RNA-Seq data obtained from TCGA[6]. The number of permutations was set to 1,000. By GSEA, we analyzed KEGG pathways to investigate possible biological functions of $E Z H 2$. The statistical criteria were: false discovery rate (FDR) $₫ 0.05$ and $P$-value $₫ 0.05$.

\subsection{Immune infiltrate analysis}

By TIMER ( https://cistrome.shinyapps.io/timer/ ), related modules were used to analyze the potential relationship between $E Z H 2$ expression and mesothelioma immune infiltration. We examined $E Z H 2$ expression in mesothelioma tissues and its relationship with the abundance of immune infiltrates by gene modules, which included CD4 T + cells, dendritic cells, B cells, CD8 + T cells, neutrophils, and macrophages. The relationship between gene expression levels and immune infiltration was also plotted.

\subsection{Statistical analysis}

Statistical analysis was performed using R (version 3.6.3) (Statistical Analysis and Visualization). For each level in the measurement data meeting the conditions that the theoretical frequency is greater than 5 and the total sample size is greater than 40 , chi-square test is used. If there are levels that do not meet the conditions that the theoretical frequency is greater than 5 or the total sample size is greater than 40 , Fisher's exact test shall be used. In the enumeration data, chi-square test was selected to meet the normal distribution, but not the normal distribution ( $P \otimes 0.05)$, and Wilcoxon rank sum test was selected. Log-rank test, Cox regression analysis and Survival curve were performed for high and low EZH2 mRNA expression according to the survival time in the database. The results were considered statistically significant at $P<$ 0.05 .

\section{Results}

\subsection{Correlation between EZH2 expressions and clinicopathologic features of mesothelioma}

Based on 86 patients downloaded from the TCGA database, we divided the patients into high expression group and low expression group according to the median $E Z H 2$, including 43 patients with high expression and 43 patients with low expression, and Patients' pathologic clinico-characteristics are shown in Table 1.

\section{Table 1}

Summary of the clinicopathological characteristics of mesothelioma patients for $E Z H 2$ expression. 
Characteristic

\begin{tabular}{|c|c|c|c|}
\hline $\mathrm{n}$ & 43 & 43 & \\
\hline Gender, n (\%) & & & 1.000 \\
\hline Female & $7(8.1 \%)$ & $8(9.3 \%)$ & \\
\hline Male & $36(41.9 \%)$ & $35(40.7 \%)$ & \\
\hline Age, n (\%) & & & 0.665 \\
\hline$<=65$ & $25(29.1 \%)$ & $22(25.6 \%)$ & \\
\hline$>65$ & $18(20.9 \%)$ & $21(24.4 \%)$ & \\
\hline Histological type, n (\%) & & & 0.381 \\
\hline Biphasic & $9(10.5 \%)$ & $13(15.1 \%)$ & \\
\hline Diffuse malignant & $3(3.5 \%)$ & $2(2.3 \%)$ & \\
\hline Epithelioid & $31(36 \%)$ & $26(30.2 \%)$ & \\
\hline Sarcomatoid & $0(0 \%)$ & $2(2.3 \%)$ & \\
\hline Residual tumor, n (\%) & & & 0.123 \\
\hline Ro & $11(32.4 \%)$ & $5(14.7 \%)$ & \\
\hline R1 & $2(5.9 \%)$ & $1(2.9 \%)$ & \\
\hline R2 & $5(14.7 \%)$ & $10(29.4 \%)$ & \\
\hline History asbestos exposure, n (\%) & & & 1.000 \\
\hline No & $7(10 \%)$ & $7(10 \%)$ & \\
\hline Yes & $27(38.6 \%)$ & $29(41.4 \%)$ & \\
\hline Pathologic stage, n (\%) & & & 0.214 \\
\hline Stage I & $2(2.3 \%)$ & $8(9.3 \%)$ & \\
\hline Stage II & $10(11.6 \%)$ & $6(7 \%)$ & \\
\hline Stage III & $23(26.7 \%)$ & $21(24.4 \%)$ & \\
\hline Stage IV & $8(9.3 \%)$ & $8(9.3 \%)$ & \\
\hline T stage, n (\%) & & & 0.081 \\
\hline T1 & $4(4.8 \%)$ & $10(11.9 \%)$ & \\
\hline T2 & $18(21.4 \%)$ & $8(9.5 \%)$ & \\
\hline T3 & $14(16.7 \%)$ & $17(20.2 \%)$ & \\
\hline
\end{tabular}


$\mathrm{N}$ stage, $\mathrm{n}(\%)$

$\begin{array}{lll}\text { N0 } & 18(22 \%) & 25(30.5 \%) \\ \text { N1 } & 6(7.3 \%) & 4(4.9 \%) \\ \text { N2 } & 17(20.7 \%) & 9(11 \%) \\ \text { N3 } & 0(0 \%) & 3(3.7 \%)\end{array}$

M stage, $n$ (\%)

Radiation therapy, n (\%)

\begin{tabular}{|c|c|c|c|}
\hline No & 31 (36.5\%) & 29 (34.1\%) & \\
\hline Yes & 11 (12.9\%) & $14(16.5 \%)$ & \\
\hline Laterality, n (\%) & & & 1.000 \\
\hline Left & $16(19.3 \%)$ & $14(16.9 \%)$ & \\
\hline Right & 27 (32.5\%) & $26(31.3 \%)$ & \\
\hline OS event, n (\%) & & & 0.016 \\
\hline Alive & $11(12.8 \%)$ & $2(2.3 \%)$ & \\
\hline Dead & 32 (37.2\%) & $41(47.7 \%)$ & \\
\hline DSS event, n (\%) & & & 0.005 \\
\hline Alive & $17(26.2 \%)$ & $5(7.7 \%)$ & \\
\hline Dead & $16(24.6 \%)$ & $27(41.5 \%)$ & \\
\hline PFI event, n (\%) & & & 0.154 \\
\hline Alive & 16 (18.6\%) & 9 (10.5\%) & \\
\hline Dead & 27 (31.4\%) & 34 (39.5\%) & \\
\hline Age, meidan (IQR) & $62(55,69.5)$ & $65(60,68)$ & 0.536 \\
\hline
\end{tabular}

Abbreviations: OS, Overall Survival; DSS, Disease Free Survival; PFI, Progression-Free Interval.

\subsection{Survival outcomes and analysis variables}


The results of survival analysis of $E Z H 2$ expression group showed that the survival time of low expression group was 26.3 (23-32.7) months; the survival time of high expression group was 13.7 (9.917.9) months. We performed statistical tests on the data of the two groups: the results of log-rank test suggested that the difference in the survival time distribution of group groups was statistically significant $(P \otimes 0.001)$. The results of Cox regression suggested that the difference in the survival time distribution of group groups was statistically significant $(P \otimes 0.001)$. The high expression group had a worse prognosis (HR=2.75, 95\% Cl: 1.68-4.52, $P \otimes 0.001)$ (Fig.1A).

We used Cox analysis to explore the relationship between EZH2 expression and OS as well as other multivariate characteristics in mesothelioma patients. Both univariate and multivariate analysis indicated that high expression of $E Z H 2$ was associated with poor prognosis in mesothelioma patients (Table 2). Univariate correlation analysis showed that $E Z H 2$ expression was significantly associated with OS ( $P]$ 0.001). Our univariate analysis showed that pathological type ( $\mathrm{HR}=5.96,95 \% \mathrm{Cl}: 0.55-64.67, P=0.020)$ and $E Z H 2$ expression ( $\mathrm{HR}=2.35,95 \% \mathrm{Cl}: 1.62-3.42, P \otimes 0.001)$ were significantly associated with OS in mesothelioma patients, and multivariate Cox regression forest plot was drawn according to the results of multivariate analysis. From the results of forest plot, it could be seen that $E Z H 2$ expression was an independent prognostic factor for the survival of mesothelioma patients $(\mathrm{HR}=2.63,95 \% \mathrm{Cl}: 1.02-6.79$, $P=0.046$ ) (Fig.1B). The distribution of $E Z H 2$ expression, survival of mesothelioma patients, and $E Z H 2$ expression are shown in Fig.1C. The expression of EZH2 had a good predictive effect on prognosis, because ROC curves showed that EZH2 expression predicted 1-year survival with an AUC of 0.740, 2-year survival with an AUC of 0.756, and 3-year survival with an AUC of 0.692 (Fig. 1D).

\section{Table 2}

Correlation between OS and multivariable characteristics in TCGA patients via Cox regression: Univariate survival model and Multivariate survival model. 
Characteristics

Total $(\mathrm{N}) \quad$ Univariate analysis

Multivariate analysis

Hazard ratio $(95 \% \mathrm{Cl}) \quad P$ value Hazard ratio $(95 \% \mathrm{Cl}) \quad P$ value

\begin{tabular}{|c|c|c|c|c|c|}
\hline Age & 85 & & & & \\
\hline$\leq 65$ & 46 & $1.27(0.81-2.09)$ & 0.286 & $1.10(0.45-2.66)$ & 0.834 \\
\hline$>65$ & 39 & & & & \\
\hline Gender & 85 & & & & \\
\hline Female & 15 & $0.94(0.52-1.73)$ & 0.850 & $0.46(0.12-1.72)$ & 0.247 \\
\hline Male & 70 & & & & \\
\hline Histological type & 58 & & & & \\
\hline Epithelioid & 57 & $13.49(1.51-120.73)$ & 0.020 & $5.96(0.55-64.67)$ & 0.142 \\
\hline Sarcomatoid & 1 & & & & \\
\hline Pathologic stage & 85 & & & & \\
\hline Stage I\&Stage II & 26 & $0.97(0.58-1.65)$ & 0.923 & $0.75(0.19-3.00)$ & 0.678 \\
\hline Stage III\&Stage IV & 59 & & & & \\
\hline T stage & 83 & & & & \\
\hline T1\&T2 & 39 & $0.96(0.59-1.55)$ & 0.852 & $0.87(0.26-2.96)$ & 0.823 \\
\hline T3\&T4 & 44 & & & & \\
\hline $\mathrm{N}$ stage & 81 & & & & \\
\hline N0\&N1 & 53 & $0.90(0.54-1.51)$ & 0.690 & $0.62(0.21-1.90)$ & 0.408 \\
\hline N2\&N3 & 28 & & & & \\
\hline M stage & 59 & & & & \\
\hline MO & 56 & $1.92(0.45-8.09)$ & 0.376 & $3.58(0.31-41.23)$ & 0.306 \\
\hline M1 & 3 & & & & \\
\hline Radiation therapy & 84 & & & & \\
\hline No & 59 & $0.70(0.41-1.18)$ & 0.174 & $0.77(0.20-3.00)$ & 0.707 \\
\hline Yes & 25 & & & & \\
\hline$E Z H 2$ & 85 & $2.35(1.62-3.42)$ & $<0.001$ & $2.63(1.02-6.79)$ & 0.046 \\
\hline
\end{tabular}




\subsection{GSEA Enrichment Analysis}

KEGG pathway analysis was performed by GSEA software to investigate the potential biological functions of $E Z H 2$. We selected the most highly enriched signaling pathways based on the normalized enrichment fraction (NES). As shown in Table 3, KEGG pathway analysis showed that there were five pathways with the strongest positive correlation with EZH2 expression: cell cycle pathway, DNA replication pathway, cell adhesion molecule pathway, primary immunodeficiency pathway, and taste conduction pathway; and five pathways with the strongest negative correlation with $E Z H 2$ expression: glycolytic gluconeogenesis pathway, drug metabolism cytochrome P450 pathway, retinol metabolism pathway, fatty acid metabolism pathway, and ribosome pathway (Fig.2). these results suggested that pathways regulating cell cycle control and fatty acid metabolism, glycolysis, and gluconeogenesis are essential in mesothelioma patients, and they are closely associated with EZH2 expression.

\section{Table 3}

Signaling pathways most significantly correlated with $E Z H 2$ expression based on their normalized enrichment score (NES) and p-value. normalized enrichment score (NES)

\begin{tabular}{lllll}
\hline \multirow{2}{*}{ positive } & ID & NES & pvalue & p.adjust \\
\hline & KEGG_CELL_CYCLE & 2.091182 & 0.001626 & 0.043625 \\
\hline & KEGG_DNA_REPLICATION & 1.987745 & 0.001799 & 0.043625 \\
\hline & KEGG_CELL_ADHESION_MOLECULES_CAMS & 1.951839 & 0.001645 & 0.043625 \\
\hline & KEGG_PRIMARY_IMMUNODEFICIENCY & 1.947583 & 0.001821 & 0.043625 \\
\hline KEGG_TASTE_TRANSDUCTION & 1.733792 & 0.003552 & 0.052474 \\
\hline negative & KEGG_GLYCOLYSIS_GLUCONEOGENESIS & -1.915402 & 0.002304 & 0.043625 \\
\hline & KEGG_DRUG_METABOLISM_CYTOCHROME_P450 & -1.958930 & 0.002342 & 0.043625 \\
\hline & KEGG_RETINOL_METABOLISM & -2.004915 & 0.002304 & 0.043625 \\
\hline & KEGG_FATTY_ACID_METABOLISM & -2.094151 & 0.002237 & 0.043625 \\
\hline & KEGG_RIBOSOME & -2.455635 & 0.002475 & 0.043625 \\
\hline
\end{tabular}

Abbreviations: NES, Normalized Enrichment Score.

\subsection{Immune infiltration}


Independent tumor-infiltrating lymphocytes are important in the prediction of OS and lymph node metastasis. Therefore, we analyzed the correlation between $E Z H 2$ expression and the level of immune infiltration in mesothelioma tissues using TIMER. These results suggest that $E Z H 2$ expression plays a key role in immune infiltration. In addition, we also sought to determine whether there is a difference in the tumor immune microenvironment between mesothelioma patients with high $E Z H 2$ levels and mesothelioma patients with low $E Z H 2$ levels. Eighty-six tumor specimens were divided into two groups according to the expression of $E Z H 2,43$ in the high expression group and 43 in the low expression group. We used the R package: GSVA package [version 1.34.0] (Hänzelmann et al., 2013) to download the gene expression profiles of the samples to determine the levels of 24 immune cells. The GSEA algorithm was applied to 24 immune cell subtypes to help assess differences in expression levels in the high EZH2 expression group and the low expression group. In Natural Killer(NK) cells, High was lower than Low, and the difference had statistical significance $(P=0.023)$; in Mast cells, High was lower than Low, and the difference had statistical significance ( $P=0.040)$; in T helper $(\mathrm{TH})$ cells, High was higher than Low, and the difference had statistical significance $(P=0.021)$; in Th17 cells, High was lower than Low, and the difference had statistical significance $(P=0.005)$; in Th2 cells, High was higher than Low, and the difference had statistical significance (P冈0.001) (Fig.3).

\section{Discussion}

Malignant mesothelioma is a rare primary cancer with a survival of less than 1 year[1]. A trimodality treatment approach combining surgery, chemotherapy, and sequential radiation therapy (RT) represents the mainstream of current mesothelioma treatment options[7]. But survival is still not improved, and prognostic biomarkers are urgently needed to guide clinical decision-making. While the TCGA database is able to obtain a large number of data from mesothelioma patients, we searched for potential new prognostic biomarkers from them based on the TCGA database.

$E Z H 2$ is the catalytic subunit of polycomb repressive complex 2 (PRC2) that represses target genes by $\mathrm{H} 3 \mathrm{~K} 27 \mathrm{me}$ [8]. In addition, highly activated $E Z H 2$ mutations are frequently found in the early stages of cancer, suggesting a role for activated PRC2 cancer drivers. Excessive EZH2 expression, which alters histone modifications, leads to an aberrant epigenetic landscape that may contribute to cancer progression[9]. Based on this, we performed the correlation between EZH2 mRNA levels and the prognosis of mesothelioma patients and immune infiltration analysis based on the TCGA database.

We collated the data obtained from the TCGA database and performed univariate and multivariate analysis of general data including age, gender, stage, pathological type, and whether they had received radiotherapy, and the results showed that high expression of $E Z H 2$ was associated with poor prognosis in mesothelioma patients. The results of survival analysis of EZH2 expression group showed that the survival time of low expression group was 26.3(23-32.7) months; the survival time of high expression group was 13.7(9.9-17.9) months. The EZH2 expression was an independent prognostic factor for the survival of mesothelioma patients ( $\mathrm{HR}=2.63,95 \% \mathrm{Cl}$ : 1.02-6.79, $\mathrm{P}=0.046)$ (Fig.1B). As shown in Fig.1A, ROC curves showed that EZH2 expression predicted 1-year survival with an AUC of 0.740, 2-year survival 
with an AUC of 0.756, and 3-year survival with an AUC of 0.692 (Fig.1D), which indicated that EZH2 expression had a good predictive effect on prognosis. To further investigate the mechanism of $E Z H 2$ action we performed further analysis and performed KEGG pathway analysis by GSEA software to investigate the potential biological function of EZH2. KEGG pathway analysis showed that there were five pathways with the strongest positive correlation with EZH2 expression: Cell cycle, DNA replication, Cell adhesion molecule, primary immunodeficiency, and taste conduction; and five pathways with the strongest negative correlation with $E Z H 2$ expression: glycolytic gluconeogenesis pathway, drug metabolism cytochrome P450 pathway, retinol metabolism pathway, fatty acid metabolism pathway, and ribosome pathway (Fig.2). These results suggest that pathways regulating cell cycle control and fatty acid metabolism, glycolysis, and gluconeogenesis are essential in mesothelioma patients, and they are closely associated with $E Z H 2$ expression.

The complex interplay between tumors and their microenvironment remains to be elucidated. Recently, it has been found that immune infiltrating components change at every tumor stage, and specific cells have an important impact on survival. As tumors progress, the density of TH cells and NK cell increases, while the density of most $T$ cells decreases. $B$ cells are a key role in the core immune network and are associated with prolonged survival, but high expression in advanced patients has a dual role in promoting tumor recurrence and progression, so they have a dual role in tumor development. Our results showed that $E Z H 2$ was closely related to the survival of mesothelioma patients. In order to explore the relationship between $E Z H 2$ and tumor immune microenvironment, we analyzed the correlation between $E Z H 2$ expression in mesothelioma tissues and immune infiltration level using TIMER. These results indicate that $E Z H 2$ expression plays an important role in immune infiltration. In addition, we also sought to determine whether there is a difference in the tumor immune microenvironment between mesothelioma patients with high EZH2 levels and mesothelioma patients with low EZH2 levels. Among them, in NK cells, High expression group was lower than low expression group $(P=0.023)$. In Mast cells, high expression group was lower than low expression group $(P=0.040)$. In TH cells, high expression group was higher than low expression group $(P=0.021)$. In Th17 cells, high expression group was lower than low expression group $(P=0.005)$. In Th2 cells, high expression group was higher than low expression group $(P$ 『0.001).

There is increasing evidence that $E Z H 2$ can not only inhibit tumor genes, but also participate in regulating collective immune homeostasis and regulating immune-related cells, especially in the development, differentiation and function of T cells[10]. EZH2 has some regulatory effects on $\mathrm{T}$ cell differentiation and epigenetic inheritance of Treg function. Pharmacological inhibition of $E Z H 2$ in human T cells using CPI1205 caused phenotypic and functional alterations in Tregs and enhanced the cytotoxic activity of Teffs. Regulating EZH2 expression in T cells could improve the antitumor response elicited by anti-CTLA-4 treatment[11]. EZH2 inhibition was found to restore the cytotoxic response of CD8 T cells in patients with systemic lupus erythematosus (SLE), reducing the incidence of infection and thus reducing death due to infection[12]. Shane et al showed that the phosphorylation status of $E Z H 2$ determines its ability to maintain anti-tumor immunity in CD8 + T memory precursor cells, and Akt-mediated EZH2 phosphorylation is a key target for enhancing anti-tumor immunotherapy strategies[13]. 
In addition, it has been shown that $E Z H 2$ is associated with tumor resistance and its inhibitors can overcome resistance to immunotherapy. $E Z H 2$ is a major driving force in cancer cellimmunoediting. It mediates immune escape by down-regulating immune recognition and activation, up-regulating immune checkpoints and generating an immunosuppressive tumor microenvironment[14]. Knockdown or inhibition of $E Z H 2$ upregulated MET expression and phosphorylation and improved cell proliferation and EGFR-TKI resistance in vitro. Inhibition of MET or PI3K/AKT elevated EZH2 levels and restored sensitivity to EGFR-TKIs. These findings suggest that the "MET-AKT-EZH2" feedback loop regulates EGFR-TKI resistance[15]. EZH2 was also highly expressed in lung cancer with positive KRAS expression, showing a positive correlation. The expression of $E Z H 2$ was positively correlated with the expression of BRAF, especially in lung squamous cell carcinoma. High expression of $E Z H 2$ and its possible synergy with KRAS and BRAF mutations[3].

$E Z H 2$ can regulate the metabolic activity of tumor cells through epigenetic regulation, which in turn affects tumor progression[16], the famous Warburg Effect indicates that cancer cells can remodel their glucose metabolism even under hypoxia[17] As can be seen from our results, the expression of $E Z H 2$ is closely related to the glycolytic gluconeogenesis pathway. In a study, Brookes et al. found that upregulation of $E Z H 2$ can increase intracellular deoxyglucose levels and induce a slight increase in mitochondrial oxidative capacity. However, cells overexpressing EZH2 may also rely on enhanced glycolysis as their main energy source. $E Z H 2$ can promote tumorigenesis and malignant progression by activating glycolysis through EAF2-HIF1a signaling axis[18]. Accumulating evidence demonstrates that $E Z H 2$, as the catalytic subunit of PRC2, also plays a key role directly or indirectly in the metabolic process of cancer cells[16]. Recent studies have shown that EZH2 expression is significantly downregulated in response to glucose deprivation in glucose-sensitive colorectal cancer cell lines, and that $E Z H 2$ knockout cells are more resistant to glucose deprivation. $E Z H 2$ deficiency upregulates glutaminase (GLS) expression and promotes glutamate production, which in turn leads to increased intracellular glutathione (GSH) synthesis and ultimately attenuates glucose deprivation-induced reactive oxygen species (ROS)mediated cell death[19].

\section{Conclusion}

High expression of $E Z H 2$ predicts a poor prognosis in mesothelioma, or can be used as a prognostic indicator and target gene in mesothelioma. The mechanism may be related to immune infiltration and metabolism, which requires further investigation.

\section{Abbreviations}

EZH2, the prognostic value of enhancer homolog 2; TCGA, the Cancer Genome Atlas; OS, Overall Survival; DSS, Disease Free Survival; PFI, Progression-Free Interval; NES, Normalized Enrichment Score; PRC2, polycomb repressive complex 2 .

\section{Declarations}




\section{Acknowledgements}

Thanks to the staff of the department of Department of Radiation Oncology, Hebei Province Cangzhou Hospital of Integrated Traditional and Western Medicine for their help in data collecting for publication.

\section{Authors' contributions}

Kui Fan and Chuanlong Zhang conceived the manuscript and analyzed the data. Kui Fan and Chuanlong Zhang wrote the manuscript and contributed equally to this work. All authors read and approved the final manuscript.

\section{Funding}

None.

\section{Availability of data and materials}

The datasets generated and/or analysed during the current study are available in the TCGA( https://portal.gdc.cancer.gov/ ) and TIMER database ( https://cistrome.shinyapps.io/timer/ ) repository. Our study conformed to the publication guidelines provided by TCGA and TIMER database. Data can be obtained from the corresponding author according to requirements of the researchers.

\section{Ethics approval and consent to participate}

The data for this study come from the TCGA database and do not require ethical approval.

\section{Consent for publication}

Not applicable

\section{Competing interests}

The authors declare that they have no competing interests

\section{ORCID}

Kui Fan https://orcid.org/0000-0002-9281-3302 
Chuan-long Zhang https://orcid.org/0000-0002-8485-5537

Bo-hui Zhang https://orcid.org/0000-0003-4309-1312

Meng-qi Gao https://orcid.org/0000-0002-1428-891X

Yunchuan-Sun https://orcid.org/0000-0002-0505-6674

\section{References}

1. Neumann, V., Rütten, A., Scharmach, M., Müller, K.-M. \& Fischer, M. Factors influencing long-term survival in mesothelioma patients-results of the German mesothelioma register. International archives of occupational and environmental health77,191-199 (2004).

2. Gan, L.et al.Epigenetic regulation of cancer progression by EZH2: from biological insights to therapeutic potential. Biomarker research6,10 (2018).

3. Fan, K.et al.Prognostic Value of EZH2 in Non-Small-Cell Lung Cancers: A Meta-Analysis and Bioinformatics Analysis. BioMed research international2020,2380124 (2020).

4. Zauderer, M. G.et al.Phase 2, multicenter study of the EZH2 inhibitor tazemetostat as monotherapy in adults with relapsed or refractory $(R / R)$ malignant mesothelioma (MM) with BAP1 inactivation. Journal of Clinical Oncology36,8515 (2018).

5. Wang, Z., Jensen, M. A. \& Zenklusen, J. C. A Practical Guide to The Cancer Genome Atlas (TCGA). Methods in molecular biology (Clifton, N.J.)1418,111-141 (2016).

6. Subramanian, A.et al.Gene set enrichment analysis: a knowledge-based approach for interpreting genome-wide expression profiles. Proceedings of the National Academy of Sciences of the United States of America102,15545-15550 (2005).

7. Brims, F. Epidemiology and Clinical Aspects of Malignant Pleural Mesothelioma. Cancers13,4194 (2021).

8. Hanaki, S. \& Shimada, M. Targeting EZH2 as cancer therapy. The Journal of Biochemistry170,1-4 (2021).

9. Bödör, C.et al.EZH2 mutations are frequent and represent an early event in follicular lymphoma. Blood122,3165-3168 (2013).

10. Huang, J.et al.Easy or Not-The Advances of EZH2 in Regulating T Cell Development, Differentiation, and Activation in Antitumor Immunity. Frontiers in immunology12,741302 (2021).

11. Goswami, S.et al.Modulation of EZH2 expression in T cells improves efficacy of anti-CTLA-4 therapy. Journal of Clinical Investigation128,3813-3818 (2018).

12. Katsuyama, E.et al.The CD38/NAD/SIRTUIN1/EZH2 Axis Mitigates Cytotoxic CD8 T Cell Function and Identifies Patients with SLE Prone to Infections. Cell Reports30,112-123.e4 (2020).

13. He, S.et al.Ezh2 phosphorylation state determines its capacity to maintain CD8+ T memory precursors for antitumor immunity. Nature communications8,2125 (2017). 
14. Kang, N.et al.EZH2 inhibition: a promising strategy to prevent cancer immune editing. Epigenomics12,1457-1476 (2020).

15. Quan, C.et al.Loss of histone lysine methyltransferase EZH2 confers resistance to tyrosine kinase inhibitors in non-small cell lung cancer. Cancer letters495,41-52 (2020).

16. Zhang, T., Gong, Y., Meng, H., Li, C. \& Xue, L. Symphony of epigenetic and metabolic regulationinteraction between the histone methyltransferase EZH2 and metabolism of tumor. Clinical epigenetics12,72 (2020).

17. Hanahan, D. \& Weinberg, R. A. Hallmarks of cancer: the next generation. Cel/144,646-674 (2011).

18. Pang, B.et al.EZH2 promotes metabolic reprogramming in glioblastomas through epigenetic repression of EAF2-HIF1a signaling. Oncotarget7,45134-45143 (2016).

19. Liu, Y.et al.Tumor-suppressive function of EZH2 is through inhibiting glutaminase. Cell death \& disease12,975 (2021).

\section{Figures}
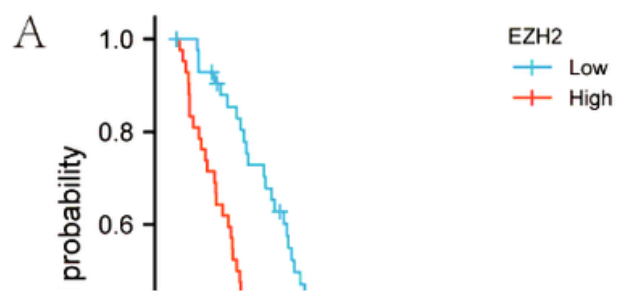

\begin{tabular}{cccc}
\hline Characteristics & Total $(\mathrm{N})$ & $\mathrm{HR}(95 \% \mathrm{Cl})$ Multivariate analysis \\
\hline Age & 85 & $1.099(0.454-2.663)$ \\
Gender & 85 & $0.456(0.121-1.723)$ \\
Histological type & 58 & $5.963(0.550-64.674)$ \\
Pathologic stage & 85 & $0.745(0.185-2.996)$ \\
T stade & 83 & $0.870(0.256-2.958)$
\end{tabular}


Figure 1

(A) Kaplan-Meier survival curves for mesothelioma patients, stratified by EZH2 expression levels.(B) Multivariate Cox analysis of expression and other clinicopathological variables. (C) EZH2 expression distribution and survival status. (D) ROC curves of EZH2.

A

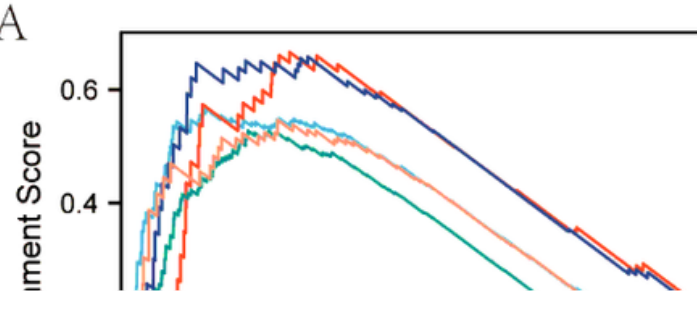

B

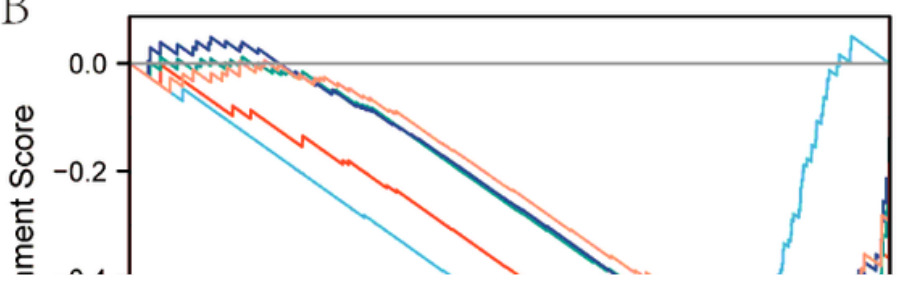

\section{Figure 2}

KEGG pathway showedfive positively correlated groups andfive negatively correlated groups.

\section{Figure 3}

(A) Correlations between $E Z H 2$ expression and immune infiltration levels (B) The varied proportions of 24 subtypes of immune cells in high and low EZH2 expression groups in tumor samples. 